\title{
Le Soudan, entre pétro-islamisme et sécession
}

Sudan - Between Petro-Islam and Secession

\section{Gérard Prunier}

\section{OpenEdition}

\section{Journals}

Édition électronique

URL : http://journals.openedition.org/transcontinentales/404

DOI : 10.4000/transcontinentales.404

ISBN : 978-2-8218-1408-0

ISSN : 1775-397X

\section{Éditeur}

Editions de la maison des sciences de l'homme

Édition imprimée

Date de publication : 30 juin 2006

Pagination : 43-55

ISBN : 2200-92169-1

ISSN : 1950-1684

\section{Référence électronique}

Gérard Prunier, "Le Soudan, entre pétro-islamisme et sécession », Transcontinentales [En ligne], 2 2006, document 4, mis en ligne le 30 septembre 2012, consulté le 07 septembre 2020. URL : http:// journals.openedition.org/transcontinentales/404; DOI : https://doi.org/10.4000/transcontinentales. 404

Ce document a été généré automatiquement le 7 septembre 2020

Tous droits réservés 


\title{
Le Soudan, entre pétro-islamisme et sécession
}

\author{
Sudan - Between Petro-Islam and Secession
}

Gérard Prunier

1 Les accords de paix signés au Kenya, à Nairobi, le 9 janvier 2005, ont été l'occasion d'un déferlement de commentaires enthousiastes qui voyaient tout à la fois dans cette cérémonie une victoire de la diplomatie classique, la liquidation d'un fantôme de la guerre froide, la " démocratisation » d'un régime islamiste et répressif et la perspective de très fructueux contrats financés tant par l'argent du pétrole que par l'aide internationale qui ne pouvait manquer de se déverser dans de brefs délais sur un pays sortant de décennies de guerre civile. Bien sûr il y avait, dans le Nord-Ouest du pays, cet ennuyeux conflit du Darfour qu'on avait résolument évité de mentionner dans l'accord de Nairobi pourtant appelé « accord de paix global » (Comprehensive Peace Agreement ou CPA), et qui laissait perplexe: des musulmans qui se tuaient entre eux, cela ne correspondait pas aux schémas que l'on croyait connaître d'une " guerre des religions " au Soudan. Mais avec la paix au Sud, la démocratie et le développement économique, on voulait croire à un retour à la normale à plus ou moins longue échéance.

2 Qu'en est-il un an plus tard? Le colonel John Garang, chef de l'Armée populaire de libération du Soudan (Sudan People's Liberation Army : SPLA) - la guérilla victorieuse qui avait signé la paix avec le gouvernement islamiste -, devenu vice-président du Gouvernement d'union nationale et président du gouvernement du Sud-Soudan, s'est tué dans un accident d'hélicoptère qui semble bien ne pas avoir été dû à un sabotage. Contrairement à des craintes trop rapidement exprimées, cette mort n'a pas affecté la situation sécuritaire, même si elle a eu d'autres effets importants sur la ligne sudiste nous y reviendrons. Ensuite, le Soudan a disparu des médias, sauf justement pour cette contrariante question du Darfour que l'on voyait bientôt réglée, mais qui s'envenimait au contraire avec les troubles débordant sur le Tchad voisin. Le reste du pays, à l'inverse, semblait opaque et silencieux, sans que l'on sache très bien si ce silence relève du calme du travail et de la reconstruction ou de celui qui précède les tempêtes. 
Pour comprendre tentons d'abord d'examiner ce qu'était l'accord de paix de janvier 2005.

\section{L'« accord de paix global » de janvier 2005}

4 Pour juger de "l'accord de paix », il faut se souvenir qu'il s'agit non pas d'un texte unique, mais d'un ensemble complexe de six documents signés entre juillet 2002 et janvier 2005, et dont seule la mise en œuvre a fait l'objet de la signature de Nairobi ${ }^{1}$.

5 Le premier document, le protocole de Machakos signé en juillet 2002, prévoit la tenue d'un référendum d'autodétermination pour le Sudqui devra être organisé à l'issue d'une période intérimaire de six années, elle-même précédée d'une période preintérimaire de six mois. La paix ayant été signée le 9 janvier 2005, le référendum doit donc être tenu le 9 juillet 2011 au plus tard.

Les arrangements de sécurité ont été signés pour leur part en septembre 2003. Leur contenu a été considérablement révisé et affiné par un second document sur le même sujet signé le 31 décembre 2004. C'est de loin le texte le plus détaillé, qui précise que pendant les six ans et demi des périodes pré-intérimaire et intérimaire, trois types de forces armées seront à l'œuvre : l'armée régulière actuelle, les forces de la SPLA, et des Unités conjointes intégrées (UCI). Ces dernières, fortes d'environ 40000 hommes, doivent être constituées pour $50 \%$ de soldats de l'armée régulière et pour $50 \%$ de guérilleros de la SPLA. Les modalités de désengagement sont très complexes. Pendant la phase 1 (les six mois de la période pré-intérimaire) les forces de la SPLA doivent évacuer l'Est du Soudan, ce qui doit représenter $30 \%$ des troupes SPLA engagées au

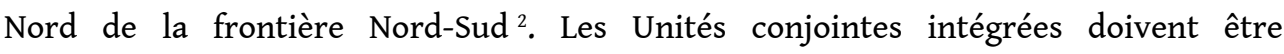
sélectionnées et entraînées, et les forces de paix de l'ONU sont déployées ${ }^{3}$. L'armée régulière commence son redéploiement vers le Nord ( $-17 \%$ dans les premiers six mois). La phase 2, devant durer du 9 juillet 2005 au 9 juillet 2008, correspond au déploiement des UCI. Pendant cette période, la SPLA évacuera l'Est, le Sud du Nil Bleu et les Monts Nouba pour y être progressivement remplacée par les UCI nouvellement créées. Le retrait des troupes gouvernementales et des forces de la SPLA est précisément planifié : la SPLA doit retirer vers le Sud $40 \%$ de ses hommes dans les huit premiers mois et $30 \%$ dans les douze mois suivants. L'armée gouvernementale doit retirer ses troupes en quatre étapes (-14\% dans les douze mois, $-19 \%$ dans les dix-huit mois suivants, $-22 \%$ dans les vingt-quatre mois suivants, et les $28 \%$ restant dans les trente derniers mois). La phase 3 ira du 9 juillet 2008 au 9 juillet 2011 et verra le déploiement complet des UCI ${ }^{4}$ ainsi que la démobilisation des troupes redondantes ${ }^{5}$. Une ultime phase 4 , de juillet à décembre 2011, devrait être marquée par le référendum d'autodétermination. Si la réponse des électeurs est favorable à l'unité du pays, les UCI seront dissoutes et toutes les forces armées seront intégrées dans un ensemble unique appelé Sudan National Armed Forces. Si au contraire la réponse va dans le sens d'une séparation du Nord et du Sud, les UCI seront dissoutes et leurs membres retourneront dans leurs régions d'origine. La question des milices, appelées Other Armed Groups, est traitée de manière succincte dans la section 11 du texte. Quinze jours après la signature de l'accord final, un Other Armed Groups Collaborative Committee aurait dû être mis sur pied, afin de régler le problème en un an. Des arrangements administratifs très complexes associant le Gouvernement d'union nationale, le gouvernement du Sud-Soudan et la communauté 
internationale ont été prévus pour assurer le respect de ces mesures et leur suivi sur le terrain.

$7 \quad$ Un accord de partage des richesses a par ailleurs été signé en décembre 2003. Il définit la manière dont les revenus de l'État doivent être répartis pendant les soixante-dix-huit mois à venir. Il contient un certain nombre de mesures concernant les questions agricoles, le fonctionnement du ministère des Finances, les douanes et les impôts. Ces deux derniers points sont secondaires, car personne au Sud ne paye d'impôts, et les aides internationales, seule ressource extérieure pour la partie méridionale du pays, seront exonérées de droits de douane. Un système bancaire islamique doit être maintenu au Nord pendant qu'un système bancaire classique doit être installé au Sud. Cela étant, tout le monde savait bien, en 2003, que la question fondamentale serait en fait celle du partage des revenus pétroliers représentant à eux seuls presque deux milliards de dollars ${ }^{6}$. Le principe d'un partage par moitié $(50 \%$ pour le Nord et $50 \%$ pour le Sud) a été retenu, mais on savait que la mise en pratique serait difficile.

8 L'accord de partage du pouvoir, signé en mai 2004, a défini la manière dont le pouvoir devait être exercé pendant les soixante-dix-huit mois à venir. Le président Omar Hassan el-Béchir resterait président de la République du Soudan. Le colonel John Garang, chef de la SPLA, deviendrait vice-président, avec un droit de veto sur les décisions du Président. Le colonel Garang serait en même temps président du gouvernement du Sud-Soudan qui allait s'installer au Sud. Pendant la période préintérimaire et les premiers mois de la période intérimaire, allant jusqu'aux élections prévues pour 2008, un gouvernement, un Parlement et une administration allaient être mis en place sur la base de la formule suivante :

- $52 \%$ des postes pour le Mutammar al-Watani (Congrès patriotique, le parti islamiste qui était précédemment le parti unique),

$10-28 \%$ pour la SPLA,

$11-14 \%$ pour les divers partis de l'opposition nordiste,

$12-6 \%$ pour les diverses forces sudistes non-membres de la SPLA.

13 Une Commission de recensement était censée définir les moyens et les procédures d'un futur recensement devant être conduit dans les trente mois ${ }^{7}$. Sur la base de ses résultats, des listes électorales doivent être établies et des élections générales (présiden-tielles, législatives et locales) organisées dans un maximum de quarante-deux mois, afin qu'un nouveau gouvernement soit mis en place.

14 Deux autres documents ont été également signés en mai 2004, les Abyei Interim Arrangements et les Nuba Mountains Interim Arrangements, qui définissent les modalités des administrations intérimaires de ces régions marginales, à la limite du Nord et du Sud, pendant la période allant jusqu'aux élections.

15 Tout cela reposait sur un extraordinaire volontarisme et sur une bonne volonté de part et d'autre qui était plus ou moins postulée par la communauté internationale et particulièrement par les États-Unis, principal artisan du processus qui avait amené la signature finale. Nulle part il n'était prévu de procédure d'arbitrage en cas de conflit entre le Gouvernement d'union nationale et l'ancien gouvernement soudanais,ni en cas de conflit de compétence entre les deux fonctions de John Garang, chef de l'exécutif du gouvernement du Sud-Soudan et vice-président du Gouver-nement d'union nationale. 


\section{En 2006, qu'en est-il de l'application de l'accord de paix global?} deux principales parties prenantes en font une lecture complètement différente. Pour l'ancien gouvernement de Khartoum, c'est un texte abstrait car tout doit être fait pour maintenir l'unité nationale. La référence à l'autodétermination fait penser aux droits de l'opposition dans la Constitution soviétique de 1936 : cela existe, c'est beau, mais il n'est pas question de trop le prendre au sérieux. Pour les Sudistes, SPLA et autres forces confondues, le protocole de Machakos est au contraire un texte de référence décisif, un vade mecum dans la voie de l'autodétermination. Cette divergence de vues a été renforcée par la mort de John Garang. Pour ce dernier, l'essentiel était d'arriver à Khartoum et de s'insérer à tout prix dans le système. Cette stratégie allait d'ailleurs provoquer un extraordinaire enthousiasme au Nord où la SPLA vit affluer, en quelques mois, des milliers de personnes, "Arabes" aussi bien qu' "Africains", qui vinrent s'enrôler dans ses rangs. Garang savait que la population nordiste était plus que lasse de la guerre, qu'elle n'aimait pas les fondamentalistes et qu'elle n'avait guère confiance dans sa propre "opposition ", usée et déconsidérée pour avoir exercé le pouvoir à de nombreuses reprises dans le passé, et l'avoir mal exercé. Il savait aussi que vivaient à Khartoum 3 millions de déplacés négro-africains qui constituaient une sorte de « lobby SPLA » tout prêt à agir ${ }^{8}$. Il se voyait donc comme une force neuve, laïque, dynamique, capable de mobiliser autour de lui une véritable coalition pré-gouvernementale. À l'en croire, les leaders de l'opposition " arabe ", vieux routiers largement discrédités de la politique nordiste, seraient incapables d'empêcher une bonne partie de l'électorat nordiste de voter pour un "nègre ${ }^{9}$ ", en dépit des préjugés raciaux de leurs aînés. Garang se voyait donc capable de gagner les élections, en réunissant autour de la SPLA une vaste coalition de Nordistes laïcs déçus, de Sudistes ambitieux et de réalistes. Ce rêve est mort avec lui, car il était le seul à avoir une carrure politique assez large et un charisme assez puissant pour assurer ce rôle de rassembleur, au-delà des préjugés raciaux et des clivages de la politique traditionnelle.

Salva Kiir Mayardit, ancien numéro deux de la SPLA, qui lui a succédé sans problème dans les jours suivant sa disparition, est un personnage très différent. Soldat de carrière très aimé des combattants de base ${ }^{10}$, un peu terne, mieux ancré que Garang dans les réalités sociales et ethniques du Sud-Soudan, il est très ignorant de la diplomatie internationale et de la politique nordiste. Il n'est pas "sécessionniste " par idéologie. Il est simplement très à l'écoute des sentiments de la base de son mouvement et de la population en général, et l'immense majorité des Sudistes est en faveur de l'indépendance de leur région. Garang entraînait sa base vers une option unitariste, et il était sans doute le seul à avoir une certaine chance de la voir réussir. Lui mort, il n'est plus question pour la masse sudiste, qui ne le suivait qu'à contrecœur dans son choix «national », de se rallier à une telle option défendue par quelqu'un d'autre. On peut donc dire que le volet « protocole de Machakos » des accords de paix du 9 janvier 2005 a pris un cours qui n'est pas celui que souhaitent le gouvernement de Khartoum ni la communauté internationale. Un climat très particulier est donc apparu à Khartoum, le Gouvernement d'union nationale donnant l'impres-sion d'être un régime en survie temporaire, un système en attente d'une rupture programmée. L'amertume et les 
tensions entre les ministres nordistes et sudistes sont constantes, même si elles se cachent sous la légendaire courtoisie soudanaise.

Les arrangements de sécurité, la partie des accords qui préoccupait le plus la communauté internationale, sont finalement ceux qui ont soulevé le moins de problème, bien qu'ils ne soient pas respectés. Très peu des mesures prévues sous ce chapitre ont en effet été mises en œuvre. La formation des Unités conjointes intégrées a pris beaucoup de retard. Ni la SPLA ni l'armée nordiste n'ont quitté les territoires qu'elles devaient évacuer selon le calendrier prévu. Quant aux milices dites "autres groupes armés ", elles sont l'objet d'un marchandage politique massif, car ce sont largement elles qui « tiennent» le Sud dans les régions non-SPLA ${ }^{11}$. Paulino Mathiep, le vieux seigneur de la guerre Nuer qui servait d'auxiliaire aux Arabes depuis 1984 dans les régions pétrolières, a fini par se rallier à la SPLA, au grand dam de l'ancien gouvernement. Certains de ses subordonnés comme Peter Gadiet ou Gordon Kong Chuol ont décidé de reprendre leur autonomie de décision et ont annoncé au contraire leur ralliement au gouvernement. La situation est en plein flux, et il faut s'attendre à voir encore de nombreux retournements dans les mois à venir. Cette paix est donc trompeuse ; l'emploi de la force demeurera l'ultime recours, pour le Nord, afin d'empêcher le Sud de faire sécession, ou pour le Sud, afin de contraindre le Nord à respecter les engagements signés. Reste à savoir si l'on attendra 2011 pour en décider ou pas. À moins de provocations énormes, les deux parties tenteront probablement de respecter les accords pour éviter de heurter la communauté internationale. Le premier qui prendra le risque de rompre l'accord de Nairobi entrera dans la guerre en perdant des points d'entrée de jeu. Mais on peut aisément imaginer des scenarii où cette considération deviendrait secondaire par rapport à un impératif vital, qu'il s'agisse de garder le pétrole pour les Nordistes ou d'arracher de force l'indépendance pour les Sudistes. Ils en rêvent depuis 1955 et ils sentent qu'elle est aujourd'hui à portée de main, inscrite dans un traité solennellement reconnu par l'ensemble de la communauté internationale, bien que celle-ci espère contre tout bon sens que les bénéficiaires ne se prévaudront pas de cette clause libératrice.

L'accord de partage du pouvoir est celui qui semble avoir été mis en œuvre de la manière la plus visible. Mais si en apparence les proportions de l'accord paraissent à peu près respectées, un examen plus approfondi montre que la réalité de l'exercice du pouvoir est différente. En effet, le SPLM ${ }^{12}$ - Sudan People's Liberation Movement - n'a eu qu'un seul ministère dit "de souveraineté ", celui des Affaires étrangères. Mais son titulaire, Lam Akol Ajawin, a vite déçu ses camarades sudistes. À peine a-t-il pris ses fonctions qu'il s'est rallié en effet aux positions arabes dominantes, au point que les Sudistes plaisantent en disant qu'il est devenu membre du Front national islamique. Il a rejeté la responsabilité de la crise au Darfour sur ceux qu'il appelle, comme Khartoum, «les rebelles ». Il a soutenu, contre tous, la présidence de l'Union africaine du général Omar el-Béchir et, ce qui a énormément choqué, il n'a pas réagi lorsque la police égyptienne a tué 258 manifestants pacifiques qui faisaient un sit-in devant les locaux du Haut Commissariat aux réfugiés au Caire en décembre 2005 pour protester contre leurs conditions de vie ${ }^{13}$. Si l'on conjugue ce retournement politique du principal ministre sudiste dans le Gouvernement d'union nationale avec l'incompétence de plusieurs autres, force est de noter que les intérêts du Sud sont bien mal défendus dans ce gouvernement «d'union nationale ». Le Mutammar al-Watani avait bien préparé les choses, et les ministres issus de la SPLA sont entourés d'une nuée d'assistants, de secrétaires, de conseillers techniques, tous arabes, qui vident la fonction ministérielle de son contenu pour la faire basculer en partie vers les sections techniques de 
l'administration et en partie vers des "cellules fantômes" créées par l'ancien gouvernement soudanais pour gérer la situation. Le président Béchir dispose en outre de douze conseillers spéciaux dont deux seulement (Bona Malwal et Mansour Khalid) n'appartiennent pas à la mouvance islamiste. Ces "conseillers spéciaux" sont en quelque sorte des répliques des ministres au sein même du Bureau de la présidence et leur influence politique est souvent plus grande que celle des ministres en titre.

Béchir dispose en outre, en la personne de Nafi Ali Nafi, ancien chef des services secrets qui garde bien en main tous ses contacts dans ce milieu très particulier, d'un « assistant spécial » dont le rôle consiste à réduire à l'impuissance le vice-président à l'échelon national, Salva Kiir. Au total les représentants du Sud sont achetés, phagocytés ou neutralisés à tous les niveaux.

L'accord de partage des richesses est à lui seul un cas particulier, étant donné le poids politique et économique de la production pétrolière. Le pétrole soudanais a été découvert entre 1979 et 1982 par la compagnie américaine Chevron dans la province du Haut Nil occidental. Mais Chevron a dû évacuer ses positions en 1984 après que les guérilleros de la SPLA eurent attaqué ses installations. La compagnie française Total, qui avait un autre grand permis d'exploitation plus au Sud, dut elle aussi se replier pour les mêmes raisons. Les Américains finirent par vendre leurs concessions en 1989 pour une bouchée de pain ${ }^{14}$ à la petite compagnie ougando-soudanaise Concorp, dirigée par un islamiste qui ne tarda pas lui-même à revendre. Les permis passèrent de propriétaires en propriétaires avant d'aboutir finalement aux mains de la Chinese National Petroleum Corporation (CNPC) qui mit tout son poids derrière un consortium destiné à achever la création d'un pipeline indispensable à la commercialisation du pétrole. La société malaisienne Petronas s'associa à hauteur de $30 \%$ avec la CNPC. La Chine ayant obtenu les crédits bancaires, un pipeline de 1,5 milliard de dollars fut construit entre 1997 et 1999, et le pétrole commença à couler vers Port-Soudan pour l'exportation. Pendant ce temps, Total ne parvenait toujours pas à tirer parti de sa concession qui demeurait enclavée loin derrière les lignes rebelles alors que les concessions rachetées par les Chinois, situées en zone gouvernementale, étaient exploitables sans attendre la fin du conflit. La production se développa rapidement et le Soudan devint en cinq ans une puissance pétrolière moyenne. Les violations des droits de l'homme liées à l'exploitation pétrolière (mitraillages de villages, nettoyage ethnique des zones d'exploration, etc.) suscitèrent des campagnes critiques à l'étranger et amenèrent peu à peu toutes les compagnies occidentales qui avaient conservé des intérêts au Soudan à les vendre. La dernière en date fut la société canadienne Talisman qui céda en 2004 ses $25 \%$ du consortium pétrolier soudanais à la compagnie d'état indienne ONGC. En 2001 le pétrole représentait déjà 590 millions de dollars en valeur à l'exportation soit $38,9 \%$ des revenus de l'État soudanais. Les niveaux actuels sont inconnus mais certainement supérieurs à 2 milliards de dollars ${ }^{15}$. 
Fig. 1

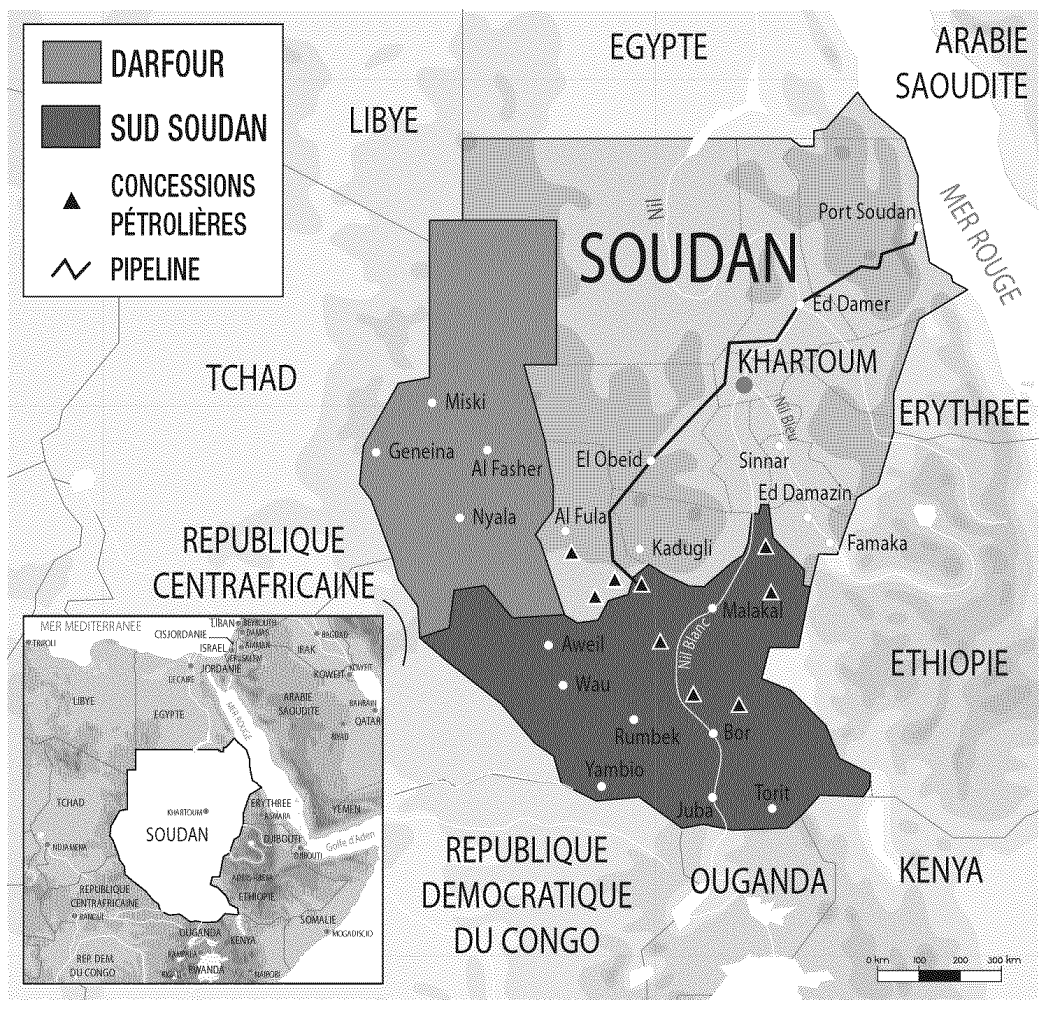

Le Soudan

Il avait été convenu que dans les soixante jours suivant la signature de l'accord de paix on créerait un système de vérification de la production pétrolière, et il était également prévu que le gouvernement soudanais expliquerait à la SPLA la nature exacte de ses accords avec les compagnies pétrolières. Un comité technique conjoint serait alors créé pour suivre les rentrées financières et mettre en place un Oil Revenue Stabilization Account (ORSA). Un prix de vente moyen devait être défini pour 2005, avec des réévaluations tous les trois mois, le prix moyen annuel étant révisé chaque année. Un système comptable devait être établi pour calculer le revenu pétrolier net, dont $2 \%$ serait alors transféré au budget de chaque province productrice ${ }^{16}$. Dans le Nord, le ministère de l'Énergie devait se charger de ce transfert. Rien de tout cela n'a été fait. Les Sudistes n'ont pu obtenir le ministère de l'Éner-gie qu'ils réclamaient: il est resté aux mains d'Awad Ahmed al-Jaz, l'un des «islamistes durs» du régime. Pour contenter la SPLA on a créé une "Commission du pétrole» d'une totale inefficacité (en trois mois elle ne s'est réunie qu'une seule fois, et la représentante de la SPLA est l'épouse de Riak Machar, un des anciens seigneurs de la guerre du Sud, dont l'absence de qualification professionnelle est patente). La commission n'a pu -définir ni le niveau de la production ni le montant des revenus qui devraient être versés au gouvernement du Sud-Soudan. Fin janvier 2006 une polémique s'est ouverte entre les éléments arabes du ministère de l'Énergie et le gouvernement du Sud-Soudan lorsque le représentant spécial des Nations unies au Soudan, Jan Pronk, a dû admettre publiquement qu'il y avait très probablement tricherie sur les fonds pétroliers. Les fonctionnaires du ministère de l'Énergie ont contre-attaqué en déclarant qu'ils avaient versé 702 millions de dollars au gouvernement du SudSoudan, mais que celui-ci ne savait qu'en faire, faute de compétences. La polémique a rebondi quand les Sudistes ont accusé Rebecca Garang, la veuve du leader de la SPLA, qui 
dirige une faction du mouvement ayant des rapports tendus avec Salva Kiir, d'avoir détourné une grande partie des sommes versées sur des comptes privés. La dis-cussion s'est - temporairement - terminée de manière peu glorieuse en mars 2006, quand le gouvernement du Sud-Soudan a dû admettre qu'il avait effectivement touché 702 millions de dollars sans pouvoir dire ce qu'il en avait fait. Cet extraordinaire aveu "d'ignorance » a du même coup relégué au second plan l'opacité du calcul des revenus pétroliers, opéré par des experts, tous nordistes. D'évidence, les mécanismes «transparents » définis dans l'accord de paix ne fonctionnent pas, et il serait nécessaire de confier l'affaire à un grand cabinet d'audit international, ce que le ministre de l'Énergie Awad Ahmed al-Jaz refuse obstinément.

\section{L'évolution de la situation au Sud et la marche à la sécession}

Sur ce point clé comme sur beaucoup d'autres, le contraste entre les mesures sophistiquées contenues dans les épais textes de l'accord de paix et les pratiques opaques et bricolées de la réalité est énorme. La première contradiction tient aux rapports inégaux entre le gouvernement soudanais signataire de l'accord et la SPLA. Le gouvernement dispose d'une expérience, de moyens humains et de moyens financiers (budgétaires et extra-budgétaires) qui sont sans commune mesure avec ceux de l'exguérilla. La SPLA ne dispose que de très peu de cadres formés et n'a en outre aucune espèce de tradition de contrôle, même vaguement « démocratique ». Garang gérait les finances du mouvement comme ses fonds propres et considérait l'administration à la manière d'un chef de bande médiéval. En dépit des décisions de la convention de Chukudum (1994) qui avait été réunie pour tenter de contrer la rébellion d'une partie du mouvement soulevé contre l'autoritarisme de Garang, la SPLA n'a jamais connu aucune forme autonome d'administration civile, et le transfert actuel de cadres militaires du mouvement vers une administration civile embryonnaire ne fonctionne guère. Les ralliements divergents des groupes composants les Forces de défense du SudSoudan (SSDF) ${ }^{17}$ ont introduit un nouveau facteur d'instabilité. En effet, ce sont souvent les milices SSDF, bien plus que les forces de la SPLA, qui contrôlent des points stratégiques clés. C'est par exemple la milice Mundari qui "couvre» la capitale sudisteJuba. C'est l'Equatoria Defence Force du Commandant Martin Kenyi qui se bat contre les guérilleros hors normes ${ }^{18}$ de la Lord's Resistance Army(LRA) ougandaise réfugiés en territoire soudanais et toujours soutenus par les services secrets de l'Armée soudanaise (Mukhabarat). Et ce sont divers groupes de miliciens Nuer qui contrôlent à 80 \% les champs pétroliers du Haut Nil occidental. Que ces milices, appelées faute de mieux SSDF, se rallient au Gouvernement d'union nationale ou au gouvernement du Sud-Soudan a de lourdes conséquences, particulièrement en termes d'enjeux pétroliers.

Autre point de délitement de l'accord de paix, la Commission de délimitation des frontières ${ }^{19}$ n'a jamais pu se réunir. Les éléments islamistes du régime souhaitent repousser la frontière vers le Sud pour grignoter des champs pétroliers en cas d'indépendance, tandis que la SPLA cherche à la remonter vers le Nord pour inclure dans le territoire devant être soumis à référendum en 2011 les fameuses "zones marginalisées » des Ingessana, des Monts Nouba et de la région d'Abyei, objet d'un débat récurrent mais mal défini entre les Nordistes, les Sudistes et la communauté internationale. La région d'Abyei, qui avait fait l'objet d'un accord séparé, a vu les 
décisions de la Commission la concernant purement et simplement rejetées par les éléments islamistes du gouvernement, sans aucune explication.

$\mathrm{Au}$ terme de cette analyse, on peut conclure à l'extrême usure du modèle national soudanais. Depuis 1956 le Soudan est considéré comme un pays à dominante arabe, avec des adjonctions africaines secondaires. Ce temps est fini, non seulement parce que le Sud a fait son entrée dans la vie nationale comme un acteur à part entière, mais aussi parce que les populations des «marges » ont fini par se rendre compte qu'elles étaient en fait la majorité. Les Arabes de la Vallée du Nil, qui ont dominé le pays sur tous les plans depuis la période coloniale, ne représentent qu'à peine $30 \%$ de la population ${ }^{20}$. Les "Arabes" des marges se rendent maintenant compte que "l'arabité » qu'ils "partagent" avec leurs frères awlad al-beled ${ }^{21}$ est une construction largement fantasmatique. Ni sur le plan des infrastructures, ni sur celui de l'éducation, ni par leur poids économique, ni par leur poids politique, ils ne peuvent jouir d'une égalité même approximative avec les habitants de la Vallée. Quant aux Noirs musulmans, ils refusent désormais de continuer à adhérer à la fausse conscience islamique qui les a maintenus dans une relation de dupes avec les awlad el-beled. Le pays craque de tous côtés et l'accord signé à Nairobi symbolise désormais la victoire de ceux qui osent se battre : la leçon ne sera pas perdue. Or les accords de paix reposent sur l'idée d'un pays binaire, " arabe » au Nord et "chrétien » au Sud. À supposer qu'il ait jamais existé, ce pays binaire n'existe plus. Il est remplacé par une marqueterie d'intérêts régionaux que les élections vont faire apparaître ouvertement, si elles ne sont pas précédées par des mouvements de guérilla. Ce mécanisme est à l'œuvre dans la guerre du Darfour, dont nous ne pouvons développer ici l'étude ${ }^{22}$ et il menace de se reproduire à l'Est du pays, dans les confins soudano-érythréens.

On constate donc une dissonance cognitive majeure entre cette réalité, et la communauté internationale qui continue à se cramponner à l'idée d'un "accord de paix global» programmatique sans vouloir admettre que personne au Soudan n'a jamais vu les choses ainsi. Pour le parti au pouvoir et la mouvance qui l'entoure, les accords de Nairobi n'ont été qu'un point de départ et nullement un point d'arrivée. L'idée de base du régime, qu'il a d'ailleurs appliquée avec beaucoup d'efficacité, est de sub-diviser les problèmes. Pour «traiter » l'opposition nordiste, il faut jouer sur les paramètres personnels, faire appel à la «solidarité arabe » et obtenir l'appui de l'Égypte qui craint une sécession du Sud et souhaite aider le gouvernement de Khartoun. Pour le Darfour, il faut découpler le règlement de la question aussi bien des processus du Nord que de l'accord de paix NordSud. Il faut ensuite entraîner la mort d'un maximum de civils pour réduire l'insurrection en " asséchant » le milieu dans lequel elle opère ${ }^{23}$. Le choix d'un règlement militaire au Darfour est dû à une idée simple, constituant l'un des principaux thèmes qui court à travers tout cet article : le Soudan est un ensemble hétérogène sur le point de craquer. Faire la paix au Sud, qui est comme un bâtiment extérieur à la maison, c'est faire la part du feu. Cette stratégie est impossible au Darfour car on ne fait pas la part du feu au cœur de sa maison. Or, vu de Khartoun, tout accord avec les rebelles du Darfour ferait craquer l'ensemble du Nord-Soudan. Il faut donc écraser la rébellion. Et pour écraser le Darfour sans que la communauté internationale n'intervienne militairement - ce qu'elle menace aujourd'hui de faire devant l'inefficacité des troupes de l'Union africaine - il faut lui jeter un os diplomatique à ronger : l'« accord de paix global » avec le Sud. Le problème, nous venons de le voir, c'est que l'accord de paix ne fonctionne pas, ou au contraire, qu'il fonctionne trop bien, mais pas dans le sens qu'espérait la communauté internationale. 

personne n'en a les moyens diplomatiques, et la SPLA n'en a pas davantage les moyens militaires. Mais certainement pas à la paix. Trop de conflits localisés ou potentiels (le Darfour n'est que le pire d'entre eux) interdisent qu'une paix globale prévale. La question est de savoir si l'on attendra 2011 pour se heurter à un état de confrontation ouverte, ou si cela se passera avant. Question corollaire d'importance: cette confrontation inévitable se réglera-t-elle par la diplomatie et par les urnes, ou par les armes ? Il est impossible de le dire à l'heure actuelle avec certitude.

Pour autant, il ne faut pas sous-estimer le gouvernement de Khartoum, quels que soient ses handicaps. De toutes les forces politiques, du Nord ou du Sud, il est la plus efficace. Il a des hommes formés, disciplinés, fanatiques pour certains, pragmatiques pour d'autres. Le pétrole lui apporte beaucoup d'argent. Ses objectifs politiques et stratégiques sont clairs. Il sait adapter ses apparentes vacillations tactiques à sa stratégie à long terme. Il dispose de moyens de patronage politique considérables et il n'a aucun scrupule d'aucune sorte. Surtout, il sait parfaitement jouer des aveuglements et des pusillanimités de la communauté internationale qu'il est passé maître dans l'art de tromper et de manipuler. Même si l'islamisme est aujourd'hui au Soudan une force minoritaire, elle n'est pas pour autant déclinante ${ }^{24}$. Le Sud est divisé ethniquement et organisationellement, mais il est passionnément uni dans sa quête d'indépendance. Le Nord a perdu ses repères, mais il tient l'argent et le pouvoir. Entre les deux, la rupture n'est plus qu'une question de temps. Reste à savoir si elle se manifestera par une « révolution de velours » à la tchécoslovaque ou par une explosion à la yougoslave.

\section{BIBLIOGRAPHIE}

- DENG F., A War of Visions, Washington DC, Brookings Institution, 1995.

- FAWZY-Rossano D., Le Soudan en question, Paris, La Table Ronde, 2002.

- HUMAN RIGHTS WATCH, Sudan, Oil and Human Rights, Washington DC, HRW, 2003.

- INTERNATIONAL CRISIS GROUP, God, Oil and Country, Bruxelles, ICG, 2002.

- JoHnson D., The Root Causes of Sudan's Civil Wars, Oxford, James Currey, 2003.

- JOK MADUT Jok: War and Slavery in the Sudan. Philadelphia, University of Pennsylvania, 2001.

- LAVERGNE M. (dir.), Le Soudan contemporain, Paris, Karthala, 1989.

- MANSOUR Khalid, War and Peace in the Sudan, Londres, Kegan Paul, 2003.

- PRUNIER G., Darfour : un génocide ambigu, Paris, La Table Ronde, 2005.

\section{ANNEXES}

De l'Égypte ottomane à l'empire britannique 
À partir de 1821, l'Égypte ottomane de Mohamed Ali commence à envahir l'espace indéfini sur son flanc sud que l'on appellera « Soudan ». En 1885, une révolte islamo-nationaliste dirigée par Mohamad Ahmed « al-Mahdi » chasse les Turco-Égyptiens du Soudan. Les Britanniques, qui ont occupé l'Égypte en 1882, reprennent les prétentions du Caire sur le Soudan, qu'ils occupent en 1898, le territoire étant alors baptisé «Condo-minium anglo-égyptien » pour maintenir la fiction d'une possession partagée. En 1916, ils occupent aussi le sultanat indépendant du Darfour. En 1924, le soulèvement nationaliste anti-britannique s'opère en coordination avec le mouvement nationaliste égyptien, qui réclame le Soudan le jour où les Anglais se retireront. Les Britanniques renoncent en 1946 à la « Southern Policy » qui les avait amenés à administrer le Sud-Soudan chrétien de manière quasiment séparée du Nord musulman. La révolution égyptienne de 1952 installe Nasser au pouvoir au Caire. Nasser reprend les prétentions de l'ex-monarchie sur le Soudan, tout en négociant avec Londres. Il obtient, en 1954, que le Soudan accède à l'indépendance en un seul bloc, c'est-à-dire sans scinder les territoires chrétiens du Sud de ceux, musulmans, du Nord. L'année suivante, à -l'annonce du départ proche des Anglais les troupes noires chrétiennes se mutinent à Torit et tuent leurs officiers arabes. Commence alors la première guerre civile.

\section{Le Soudan indépendant : cinquante ans de troubles}

1956 Le Soudan accède à l'indépendance.

1958 Coup d'État militaire du général Abboud.

1963 La rébellion sudiste prend de l'ampleur.

1964 Un soulèvement civil renverse la dictature militaire.

1965 Sadiq al-Mahdi gagne les élections et renforce la répression au Sud.

1969 Le général Jaafar al-Nimeyri, s'empare du pouvoir avec l'aide du Parti communiste en promettant une « solution socialiste » au conflit avec le Sud.

1971 Échec du coup d'État communiste contre Nimeyri. Le parti est démantelé dans le sang.

1972 Nimeyri négocie à Addis-Abeba un accord de paix avec la rébellion. Le Sud-Soudan reçoit un statut semi-autonome avec une capitale à Juba.

1979 Début de l'exploration pétrolière au Sud-Soudan par la compagnie américaine Chevron. Total prend un vaste permis au sud de celui de Chevron.

1980 Pour garder le contrôle du pétrole, Nimeyri démantèle l'autonomie sudiste.

1983 Nouveau soulèvement sudiste. Le colonel John Garang fonde en Éthiopie la Sudan People's Liberation Army (SPLA), liée au régime communiste du colonel Menguistu.

1984 Les compagnies pétrolières occidentales évacuent le Soudan.

1985 Affaibli par la guerre, la famine due à la sécheresse et la crise économique, le régime de Nimeyri est renversé par une insurrection civile.

1986 Sadiq al-Mahdi remporte les élections et poursuit la guerre au Sud. Première guerre civile du Darfour, largement causée par les Libyens auxquels Sadiq avait promis la province en échange de leur appui financier.

1989 Coup d'État islamiste du général Béchir, qui gouverne sous la tutelle de Hassan al-Tourabi, chef des Frères Musulmans. Une conférence régionale au Darfour rétablit la paix indépendamment des événements de Khartoum. 
1991 La chute du régime communiste en Éthiopie affecte la puissance militaire de la SPLA.

1993 Le colonel Garang se rapproche du président ougandais Yoweri Museveni qui lui sert

d'intermédiaire pour entrer dans les bonnes grâces des États-Unis.

1994 Les troubles reprennent au Darfour, opposant tribus noires et Arabes.

1995 La Chine s'active au Soudan : livraisons d'armes au Nord, projets pétroliers au Sud.

1999 L'oléoduc reliant les champs pétroliers du Haut Nil à Port-Soudan est achevé. La Chine fonde la Greater Nile Petroleum Corporation avec le malaisien Petronas.

2002 Épuisés par la guerre civile, le régime islamiste et la SPLA conviennent d'un cessez-le-feu et entament des conversations de paix.

2003 Se jugeant marginalisés par les négociations entre le oguvernement et la SPLA, les tribus noires du Darfour se révoltent contre Khartoum.

2004 Massacres d'ampleur génocidaire au Darfour, organisés par Khartoum qui utilise des milices arabes pour tuer les civils noirs.

2005 Signature d'une paix dite « globale « entre la SPLA et Khartoum, mais dont le Drafour est exclu.Mort accidentelle du colonel John Garang six mois plus tard. L guerre se poursuit au Darfour.

2006 Signature le 5 mai, d'un accord entre Kharoum et une faction des insurgés di Darfour.

\section{NOTES}

1. Ce calendrier de mise en œuvre est extrêmement précis et fait 101 pages.

2. Les 6000 hommes du SPLA présents en Érythrée constituent un point particulier, car rien n'a été dit de leur statut qui cause aujourd'hui un nouveau et grave problème.

3. Il n'y a toujours, à l'heure actuelle, qu'environ 6000 hommes déployés sur les 10000 prévus initialement. Aucun n'est déployé au Darfour.

4. Autre point obscur de la section 18 du texte : en deux endroits différents de la même section, le déploiement complet des Unités conjointes intégrées est prévu en vingt et un mois et, plus loin, en cinquante-deux mois.

5. Autre incertitude : si les forces UCI sont bien définies, il n'y a nulle par de chiffre global des forces gouvernementales ni des forces de la SPLA. Le nombre des soldats à payer (et de ceux à démobiliser plus tard) n'est pas non plus déterminé.

6. Trois milliards au cours actuel du baril et au niveau actuel de la production.

7. Le dernier recensement date de 1983.

8. Ce sont eux qui se sont soulevés en août 2005 après la mort de Garang, et qui ont tué des civils nordistes avant d'être eux-mêmes victimes de la revanche des milices arabes.

9. Au Soudan on dit 'abd, « un esclave ».

10. Ce n'était pas le cas de Garang, paradoxalement plus populaire aux yeux des étrangers qu'à ceux de ses propres troupes, qui renâclaient contre sa " gestion à distance » de la guérilla.

11. L'armée régulière nordiste demeure cantonnée dans les grandes villes et se risque peu en dehors d'elles.

12. Le SPLM est le double politique de la SPLA. C'est sous ce nom qu'est formulé le programme politico-économique du mouvement sudiste. La symbiose est telle entre les deux organisations qu'on en trouve parfois mention sous le sigle combiné SPLA/M ou SPLA/SPLM. John Garang, à la tête de la SPLA, était aussi président du SPLM. (NDLR) 
13. La première protestation aurait bien sûr dû concerner le nombre des victimes, faussement estimé par les autorités égyptiennes à 28 morts. Les 258 personnes décédées ont été dénombrées par les organisations de réfugiés dans les morgues de plusieurs hôpitaux du Caire.

14. Les permis furent vendus 22 millions de dollars alors que Chevron avait dépensé 1,3 milliard de dollars entre 1979 et 1984 . Mais l'opération permit au pétrolier américain de récupérer 900 millions de dollars du service des impôts américains pour « pertes en opération ».

15. Comme nous allons le voir, il y a de graves divergences de vues sur la production soudanaise et sur l'argent qui en est tiré.

16. Les divisions administratives régionales sont appelées « états » au Soudan.

17. Southern Sudan Defence Forces. Ce sigle est artificiel car on compte en fait dix-huit milices distinctes, de taille extrêmement variable, mais toutes constituées sur des bases étroitement ethniques.

18. L'Armée de la résistance du Seigneur entend fonder un gouvernement basé sur les dix commandements bibliques.

19. Par ce terme, il faut entendre la frontière intérieure Nord-Sud, et non les frontières internationales. La frontière Nord-Sud grossièrement acceptée aujourd'hui est celle qu'avait déterminée -l'administration britannique en 1926 et qui avait été confirmée à l'indépendance en 1956, ainsi que lors de la paix mettant fin à la première guerre civile en 1972. Elle est aujourd'hui remise en cause tant par les Arabes que par la SPLA, mais dans des sens opposés.

20. La population totale du Soudan était évaluée à 35 millions d'habitants en 2004, pour une superficie de 2,5 millions de $\mathrm{km}^{2}$. (NDLR)

21. Les Arabes de la Vallée du Nil définissent par ce terme, qui veut dire mot à mot « les enfants du pays ", leur communauté. L'expression est implicitement raciste, car elle implique que les Sudistes ne sont pas des citoyens et que les Arabes non-riverains du Nil ne le sont pas vraiment non plus.

22. Sur le conflit du Darfour, voir Gérard Prunier, Le Darfour : un génocide ambigu, Paris, La Table Ronde, 2005.

23. De ce point de vue et pour une étude sur la démographie différentielle des groupes ethniques comme cause de la guerre du Darfour, voir l'excellent travail d'Éric Denis: "Démographie ethnique, modernisation et violence au Darfour », Les Annales d'Ethiopie, XXI, 2005, pp. 91-135.

24. L'affairisme du pouvoir a aliéné ceux des islamistes qui étaient des idéologues convaincus. Ils se regroupent aujourd'hui derrière al-Tayeb Moustafa, oncle du président Béchir et chef du Mouvement pour une paix juste qui prône l'abandon du Sud, pétrole compris, pour reconstruire le Nord sous la forme d'une République islamique pure et dure. Ses vues sont minoritaires, mais attirent une forte attention dans le climat actuel.

\section{RÉSUMÉS}

Très détaillé, l'accord de paix signé le 9 janvier 2005 pour mettre fin à la guerre civile soudanaise vieille de plus de vingt ans a été salué comme un triomphe diplomatique mais son application connaît de nombreux contretemps. Par-delà d'inévitables difficultés pratiques, ces contretemps peuvent généralement être attribués à la mauvaise volonté des éléments de l'ancien gouvernement de Khartoum dominant l'actuel Gouvernement d'unité nationale issu de l'accord de paix. Un examen des différentes questions en jeu amène à conclure que, si les choses 
continuent de cette manière, un vote d'indépendance des populations du Sud lors du référendum d'autodétermination prévu pour juillet 2011 est extrêmement probable.

The comprehensive peace agreement that ended the 20-year Sudanese civil war, signed in Nairobi on January 9 2005, was universally hailed as a diplomatic triumph. However, its implementation has been marred by numerous problems. Apart from the inevitable practical difficulties, most of these issues can be attributed to deliberate resistance on the part of former members of the previous Government of Sudan who now dominate the new Government of National Unity set up under the peace agreement. The paper examines these problems and concludes that, if this situation continues, the Southern Sudanese will very probably vote for independence in the self-determination referendum planned for July 2011.

\section{INDEX}

Index géographique : Soudan, Sud Soudan

Keywords : Sudan, civil war, peace agrement, South Sudan

Mots-clés : accord de paix, guerre civile

\section{AUTEUR}

\section{GÉRARD PRUNIER}

Gérard Prunier est chercheur au CNRS et dirige actuellement le Centre français d'études éthiopiennes à Addis-Abeba. Il est l'auteur de La question indienne en Ouganda (1896-1972) Paris, ADPF, 1990, The Uganda Crisis (1959-1994) : History of a Genocide, Londres et New York, Hurst/Columbia University Press, 1995 et Darfour : un génocide ambigu, Paris, La Table ronde, 2005. Il a aussi dirigé des ouvrages collectifs sur le Kenya, l'Ouganda et -l'Éthiopie, dont le prochain (L'Éthiopie contemporaine) paraîtra aux Éditions Karthala en 2006.

cfee@telecom.net 